\title{
Automatic design of morphological operators
}

\author{
JUNIOR BARRERA \\ Departamento de Ciência da Computação. \\ instituto de Matemática e Estatística. \\ Universidade de São Paulo. São Paulo, SP, Brazil. JB@ime.usp.br
}

\author{
GERALD J. F. BANON \\ Divisão de Processamento de Imagens. \\ Instituto Nacional de Pesquisas Espaciais. \\ SÃo José dos Campos, SP, Brazil. BANON@DPI.INPE.BR \\ EDWARd R. Dougherty \\ Department of Electrical Engeneering. \\ Texas A \& M University. \\ College Station, TX, USA. Edward@ee.tamu.edu
}

\begin{abstract}
August 30, 2002
Abstract. A central paradigm in mathematical morphology is the decomposition (i.e., representation) of lattice operators (i.e., mappings) in terms of four classes of elementary operators: dilations, erosions, anti-dilations and anti-erosions. The result of the decomposition of a lattice operator in terms of these elementary operators is called a morphological operator. Any lattice operator can be represented by a canonical morphological operator: the union of morphological sup-generating operators (i.e., intersection of an erosion and an anti-dilation). The sup-generating operators of this decomposition are parameterized by a set of maximal intervals, called operator basis. The automatic design of morphological operators consists of estimating the operator basis from a sequence of examples (i.e., input-ideal output pairs). This technique has been applied to solve several image and signal processing poroblems.
\end{abstract}

\section{INTRODUCTION}

A central paradigm in mathematical morphology is the decomposition (representation) of complete lattice operators ( mappings) in terms of four classes of elementary 
operators: dilations, erosions, anti-dilations and anti-erosions. The rules for performing these representations can be described as a formal language, the morphological language [4]. The vocabulary of this language is composed of the four classes of elementary operators and the lattice operations of intersection and union. A phrase of the morphological language is called a morphological operator.

The adequacy of morphological operators to solve image and signal processing problems show that the morphological language is expressive, meaning that many useful operators can be represented by relatively few words. The completeness of the morphological language was first studied by Matheron in 1975, in his classical book, Random Sets and Integral Geometry [35], p.219). In the context of translation invariant set operators (operators that commute with translation), Matheron introduced the notion of the operator kernel, which is a family of sets whose images by the operator cover the origin. He proved that any increasing (i.e., inclusion preserving) and translation invariant set operator can be represented by a union of erosions, with structuring elements in the operator kernel. Ten years later, in 1985, Maragos [33], and Dougherty and Giardina [16], independently, proved that Matheron's decomposition result could be simplified in the sense that just a minimal subset of the kernel, called the operator basis, is enough to perform the decomposition. Maragos included a topological condition for the existence of the basis, that operator to be decomposed be upper semicontinuous under the hit-miss topology. Maragos extended these results to function operators [34].

Some years later, in 1991, Banon and Barrera proved that any translation invariant set operator could be decomposed as the union of sup-generating operators (i.e., intersections of erosions and anti-dilations), with structuring elements that are extremities of intervals included in the operator kernel [2]. Banon and Barrera also proved the existence of a basis for the family of upper semi-continuous operators under the hit-miss topology. The results of Matheron, Maragos, and Dougherty and Giardina for increasing set operators are particular cases of the general result. From a suggestion of Matheron, Banon and Barrera also applied their decomposition to operators that can be built as the intersection of an increasing and a decreasing operator (i.e., an operator that inverts the inclusion relation).

A couple of years later, in 1993, Banon and Barrera found the notion of morphological connection, which extends the notion of Galois connection. While a Galois connection induces an isomorphism between the set of dilations and erosions, the morphological connection induces an isomorphism between the set of morphological sup-generating operators and a set formed by the Cartesian product of dilations and anti-dilations. This property and the generalization of the notion of kernel are the keys to finding a constructive representation for any lattice operator in terms of a union of sup-generating operators. This decomposition also admits a simplified form parameterized by the operator basis. All previous decomposition results become 
particular cases of this general decomposition of lattice operators [3].

A remarkable property of the lattice-operator decomposition is that it applies for both continuous and discrete lattices. The decomposition of discrete operators that are both translation invariant and locally defined, meaning the value of a transformed signal in a given point depends just of a neighborhood of this point in the input signal, permits the development of a technique for the design of morphological operators based on the observation of examples of the desired transformation, the sequence being of pairs formed from the input and the desired ideal output. This technique consists essentially in the estimation of the basis of an operator that minimizes a given statistical error measure. After about a decade of research Dougherty, Barrera and their collaborators have obtained theoretical and applied results that prove the adequacy of discrete techniques for solving many image and signal processing problems [12], [8], [10], [14]. A salient example is the design of debluring filters that have better performance than the classical optimal Wiener filter [27]. Two points are at issue here. First, the Wiener filter is optimal among linear filters, and linear filters cannot provide sufficient restoration for many types of blur. Second, the morphological deblurring operators are not increasing, which is what gives them the ability to perform optimal nonlinear deblurring.

Following this introduction, Section 2 recalls the canonical decomposition of set $\mathrm{W}$-operators. Section 3 recalls the design of set W-operators. Section 4 recalls the morphological and loose canonical decompositions of lattice operators. Section 5 presents applications of the canonical lattice decompositions to gray-scale image operators design. Finally, Section 6 discusses the impact of this research and shows some future perspectives of the field.

\section{Set W-operator Decomposition}

In this section, we present the family of set $\mathrm{W}$-operators and give their representation, in terms of sup-generating operators, and characterization, in terms of Boolean functions.

2.1. Set $\mathbf{W}$-operator. The set $E$ is assumed to be an Abelian group with respect to a binary operation denoted + . The zero element of $(E,+)$ is denoted by $o$. This zero element is also called the origin of $E$.

Let $X^{t}$ denote the transpose of a subset $X$ of $E$, that is, $X^{t} \triangleq\{y \in E: y=$ $-x, x \in X\}$.

For any $h \in E$ and $X \subseteq E$, the set $X_{h} \triangleq\{x \in E: x-h \in X\}$ is called the translation of $X$ by $h$. In particular, $X_{o}=X$.

Let $\mathcal{P}(E)$ denote the power set of $E$. A set operator is any mapping defined from $\mathcal{P}(E)$ into itself. The set $\operatorname{Fun}(\mathcal{P}(E), \mathcal{P}(E))$ of all the operators from $\mathcal{P}(E)$ to $\mathcal{P}(E)$ inherits the complete lattice structure of $(\mathcal{P}(E), \subseteq)$ on setting, $\forall \Psi_{1}, \Psi_{2} \in$ 
$\operatorname{Fun}(\mathcal{P}(E), \mathcal{P}(E)), \Psi_{1} \leq \Psi_{2} \Leftrightarrow \Psi_{1}(X) \subseteq \Psi_{2}(X), \forall X \in \mathcal{P}(E)$. The supremum and infimum of a subset $\mathcal{T}$ of the complete lattice $(\operatorname{Fun}(\mathcal{P}(E), \mathcal{P}(E)), \leq)$ verify $(\bigvee \mathcal{T})(X)=\bigcup\{\Upsilon(X): \Upsilon \in \mathcal{T}\}$ and $(\bigwedge \mathcal{T})(X)=\bigcap\{\Upsilon(X): \Upsilon \in \mathcal{T}\}, \forall X \in \mathcal{P}(E)$

A set operator $\Psi$ is called translation invariant (t.i.) if and only if (iff), $\forall h \in E$, $\Psi\left(X_{h}\right)=\Psi(X)_{h}, \forall X \in \mathcal{P}(E)$.

Let $W$ be a finite subset of $E$. A set operator $\Psi$ is called locally defined on $W$ iff, $\forall h \in E, h \in \Psi(X) \Leftrightarrow h \in \Psi\left(X \cap W_{h}\right), \forall X \in \mathcal{P}(E)$.

Let $\mathcal{T}_{W}$ denote the collection of t.i. operators locally defined on $W$. The elements of $\mathcal{T}_{W}$ are called set $W$-operators or, simply, $W$-operators. The pair $\left(\mathcal{T}_{W}, \leq\right)$ constitutes a sublattice of the lattice $(\operatorname{Fun}(\mathcal{P}(E), \mathcal{P}(E)), \leq)$.

2.2. Representation. The kernel $\mathcal{K}(\Psi)$ of a $W$-operator $\Psi$ is the subcollection of $\mathcal{P}(W)$ defined by $\mathcal{K}(\Psi) \triangleq\{X \in \mathcal{P}(W): o \in \Psi(X)\}$.

Proposition 1. The mapping $\mathcal{K}$ from $\mathcal{T}_{W}$ to $\mathcal{P}\left(\mathcal{P}(W)\right.$ ) defined by, for any $\Psi \in \mathcal{T}_{W}$,

$$
\mathcal{K}(\Psi) \triangleq\{X \in \mathcal{P}(W): o \in \Psi(X)\}
$$

constitutes a lattice isomorphism between the lattices $\left(\mathcal{T}_{W}, \leq\right)$ and $(\mathcal{P}(\mathcal{P}(W)), \subseteq)$. The inverse of the mapping $\mathcal{K}$ is the mapping $\mathcal{K}^{-1}$ defined by, for any $\mathcal{X} \subseteq \mathcal{P}(W)$ and $X \in \mathcal{P}(E)$,

$$
\mathcal{K}^{-1}(\mathcal{X})(X) \triangleq\left\{x \in E: X_{-x} \cap W \in \mathcal{X}\right\}
$$

As a consequence of the last proposition, the following equalities hold: for any $\Psi_{1}, \Psi_{2} \in \mathcal{T}_{W}, \mathcal{K}\left(\Psi_{1} \wedge \Psi_{2}\right)=\mathcal{K}\left(\Psi_{1}\right) \cap \mathcal{K}\left(\Psi_{2}\right)$ and $\mathcal{K}\left(\Psi_{1} \vee \Psi_{2}\right)=\mathcal{K}\left(\Psi_{1}\right) \cup \mathcal{K}\left(\Psi_{2}\right)$

The set operator $\Gamma$, defined by $\Gamma(X)=X^{c}, \forall X \in \mathcal{P}(E)$, is called negation. Let $A, B \in \mathcal{P}(E)$. The operations

$$
A \oplus B \triangleq \bigcup\left\{A_{b}: b \in B\right\} \text { and } A \ominus B \triangleq \bigcap\left\{A_{-b}: b \in B\right\}
$$

are called, respectively, Minkowski addition and subtraction. Let $B \in \mathcal{P}(E)$. The t.i. set operators $\Delta_{B}$ and $E_{B}$ defined by $\Delta_{B}(X)=X \oplus B$ and $E_{B}(X)=X \ominus B$, for any $X \in \mathcal{P}(E)$, are called, respectively, dilation and erosion by $B$. The parameter $B$ that characterizes a dilation or an erosion is called a structural element or a structuring element .

Let $A, B \in \mathcal{P}(W)$ such that $A \subseteq B$. The t.i. set operator $\Lambda_{[A, B]}$ defined by, for any $X \in \mathcal{P}(E)$,

$$
\Lambda_{[A, B]}(X) \triangleq\left\{x \in E: A \subseteq X_{-x} \cap W \subseteq B\right\}
$$


is called sup-generating operator. This operator was first stated by Serra ([42], p.39) in the form $\Lambda_{\left[A, B^{c}\right]}$ and called hit-miss operator.

Note that $\Lambda_{[A, B]}$ is locally defined on $W$ and can be, equivalently, represented by, for any $X \in \mathcal{P}(E)$,

$$
\Lambda_{[A, B]}(X)=E_{B}(X) \cap \Gamma \Delta_{B^{t c}}(X),
$$

where the complement of $B$ is taken relative to $W$.

Given $A, B \in \mathcal{P}(W)$, the subcollection $[A, B]$ of $\mathcal{P}(W)$ defined by

$$
[A, B] \triangleq\{X \in \mathcal{P}(W): A \subseteq X \subseteq B\}
$$

is called an interval.

Proposition 2. The kernel of a sup-generating operator is an interval, that is,

$$
\mathcal{K}\left(\Lambda_{[A, B]}\right)=[A, B]
$$

Theorem 3. If $\Psi$ is a $W$-operator, then, for any $X \in \mathcal{P}(E)$,

$$
\Psi(X)=\bigcup\left\{\Lambda_{[A, B]}(X):[A, B] \subseteq \mathcal{K}(\Psi)\right\}
$$

Proof. $\quad \mathcal{K}(\Psi)=\bigcup\{[A, B] \subseteq \mathcal{P}(W):[A, B] \subseteq \mathcal{K}(\Psi)\}$, since any subset of a complete lattice can be built by the union of its intervals; thus, $\mathcal{K}(\Psi)=\bigcup\left\{\mathcal{K}\left(\Lambda_{[A, B]}\right)\right.$ : $[A, B] \subseteq \mathcal{K}(\Psi)\}$, by Proposition 2 , and $\Psi=\bigvee\left\{\Lambda_{[A, B]}:[A, B] \subseteq \mathcal{K}(\Psi)\right\}$, since $\mathcal{K}$ is a lattice isomorphism between $\left(\mathcal{T}_{W}, \leq\right)$ and $(\mathcal{P}(\mathcal{P}(W)), \subseteq)$.

The decomposition of the last theorem is called canonical sup-decomposition. Though this decomposition is quite general, it may lead to inefficient computational representation for most $\mathrm{W}$-operators, in the sense that a smaller family of sup-generating operators may be sufficient to represent the same operator.

An interval $[A, B]$ is called maximal in a collection of intervals $\mathbf{I}$ iff, $[A, B] \in \mathbf{I}$ and, $\forall\left[A^{\prime}, B^{\prime}\right] \in \mathbf{I},[A, B] \subseteq\left[A^{\prime}, B^{\prime}\right]$ implies that $[A, B]=\left[A^{\prime}, B^{\prime}\right]$. The set $\mathbf{B}(\Psi)$ of all maximal intervals contained in $\mathcal{K}(\Psi)$ is called basis of $\Psi$.

Theorem 4. If $\Psi$ is a $W$-operator, then, for any $X \in \mathcal{P}(E)$,

$$
\Psi(X)=\bigcup\left\{\Lambda_{[A, B]}(X):[A, B] \in \mathbf{B}(\Psi)\right\}
$$


Proof. Once $\mathrm{W}$ is finite, we can express $\mathcal{K}(\Psi)$ in terms of its maximal intervals, that is, $\mathcal{K}(\Psi)=\bigcup\left\{\mathcal{K}\left(\Lambda_{[A, B]}\right):[A, B] \in \mathbf{B}(\Psi)\right\}$. The result follows by the same arguments used to prove Theorem 3.

We should observe that there are also dual representations in terms of the kernel and basis of a W-operator. These dual representations are called inf-canonical, since they are the intersection of inf-generating operators (i.e., dual of sup-generating operators).

In particular, when the set $\mathrm{W}$-operator $\Psi$ is increasing $[A, B] \in \mathbf{B}(\Psi)$ implies that $B=W$ and the canonical sup-decomposition reduces to an union of erosions, that is, for any $X \in \mathcal{P}(E)$,

$$
\Psi(X)=\bigcup\left\{E_{A}(X): A \in \mathcal{B}(\Psi)\right\},
$$

$\mathcal{B}(\Psi)=\{A:[A, B] \in \mathbf{B}(\Psi)\}$. This last representation is exactly the one proposed by Matheron in ([35], p.219).

2.3. Characterization. We have seen that W-operators can be represented by their kernel or basis. We will study now a third way of representing W-operators: the equivalent Boolean function.

Let $T$ be the mapping defined from $\mathcal{T}_{W}$ to $\operatorname{Fun}(\mathcal{P}(W),\{0,1\})$ defined by, for any $X \in \mathcal{P}(E)$,

$$
T(\Psi)(X) \triangleq \begin{cases}1 & \text { if } o \in \Psi(X) \\ 0 & \text { otherwise }\end{cases}
$$

The mapping $T$ constitutes a lattice isomorphism between the complete lattices $\left(\mathcal{T}_{W}, \leq\right)$ and $(\operatorname{Fun}(\mathcal{P}(W),\{0,1\}), \leq)$ and its inverse $T^{-1}$ is defined by, for every $\psi \in$ $\operatorname{Fun}(\mathcal{P}(W),\{0,1\})$, for any $X \in \mathcal{P}(E)$,

$$
T^{-1}(\psi)(X) \triangleq\left\{x \in E: \psi\left(X_{-x} \cap W\right)=1\right\} .
$$

3. Set W-Operator Design

The morphological representation theory provides a natural framework for automatic operator design. An operator is desired to optimally estimate an image when it is observed after going through a system.

3.1. Unconstrained design. To frame the problem, binary digital images are modeled as discrete random sets. The task is to design an operator $\Psi$ so that, given an observed random set $S, \Psi(S)$ is probabilistically close to a desired (ideal) random set $I$. The closeness of the ideal and the estimator $\Psi(S)$ is measured by some probabilistic error measure $\operatorname{Er}[I, \Psi(S)]$. Assuming the operator belongs to 
some family $\mathcal{I}$, an optimal operator relative to $\mathcal{I}$ is an operator $\Psi_{\text {opt }} \in \mathcal{I}$ for which $\operatorname{Er}\left[I, \Psi_{\text {opt }}(S)\right] \leq \operatorname{Er}[I, \Psi(S)]$, for all $\Psi \in \mathcal{I}$. If every operator $\Psi \in \mathcal{I}$ has a representation, then optimization can be viewed as finding the representation defining an operator possessing minimum error $\operatorname{Er}\left[I, \Psi_{\text {opt }}(S)\right]$.

When $\mathcal{I}$ is the family of set W-operators, estimation of $I$ from $S$ by a W-operator $\Psi$ requires finding a Boolean function $\psi$ to minimize error. Since, $\Psi$ is translationinvariant, we make the modeling assumption that $I$ and $S$ are jointly strict sense stationary. Hence, if $\mathbf{X}$ is the random vector of binary values in the translate of $W$ to $z$ and $Y \triangleq I(z)$, then the joint probability distribution for $\mathbf{X}$ and $Y$ is independent of $z$, so that estimating $Y$ from $\mathbf{X}$ yields a translation invariant operator. We denote random variables and random vectors by upper-case italic and bold face letters, respectively. Realizations of the random variable $Y$ and the random vector $\mathbf{X}$ will be denoted by $y$ and $\mathbf{x}$, respectively.

For operator optimization we require a loss function $l:\{0,1\}^{2} \rightarrow[0, \infty)$, where $l(a, b)$ measures the cost of the difference between $a$ and $b$, with $l(0,0)=l(1,1)=0$. Relative to the loss function (and owing to stationarity), filter error, $\operatorname{Er}(\Psi)$, is given by the expected loss from estimating $Y \triangleq I(z)$ by $\psi(\mathbf{X}) \triangleq \Psi(S)(z)$, where $z$ is an arbitrary pixel:

$$
\begin{aligned}
& \operatorname{Er}(\Psi) \triangleq E\left[l(Y, \psi(\mathbf{X})]=\sum_{\{\mathbf{x}: \psi(\mathbf{x})=0\}} l(1,0) P(Y=1 \mid \mathbf{x}) P(\mathbf{x})\right. \\
& +\sum_{\{\mathbf{x}: \psi(\mathbf{x})=1\}} l(0,1) P(Y=0 \mid \mathbf{x}) P(\mathbf{x})
\end{aligned}
$$

where $P(\mathbf{x})$ denotes $P(\mathbf{X}=\mathbf{x})$. An optimal operator is one whose Boolean function $\psi$ minimizes $\operatorname{Er}(\Psi)$. Although there can be more than one operator achieving minimal error, we shall denote "the" optimal operator and its Boolean function by $\Psi_{\text {opt }}$ and $\psi_{\text {opt }}$, respectively, the convention being that, from the optimization view point, operators having minimal error are equivalent.

The mean-absolute error (MAE) loss function is defined by $l(y, \psi(\mathbf{x}))=|y-\psi(\mathbf{x})|$. Since $y$ and $\psi(\mathbf{x})$ are binary-valued, the loss function is given by $l(1,0)=l(0,1)=1$ and $l(0,0)=l(1,1)=0$. An optimal operator is determined by $\psi_{\text {opt }}(\mathbf{x})=1$ if $P(Y=1 \mid \mathbf{x})>0.5$ and $\psi_{\text {opt }}(\mathbf{x})=0$ if $P(Y=1 \mid \mathbf{x}) \leq 0.5$. The MAE for an optimal operator is given by

$$
M A E\left[\Psi_{\text {opt }}\right]=\sum_{\{\mathbf{x}: \psi(\mathbf{x})=1\}} P(Y=0 \mid \mathbf{x}) P(\mathbf{x})+\sum_{\{\mathbf{x}: \psi(\mathbf{x})=0\}} P(Y=1 \mid \mathbf{x}) P(\mathbf{x})
$$

If a suboptimal operator $\Psi$ is used instead of $\Psi_{\text {opt }}$, then there is an increase in error. To quantify the error increase, if $P(\mathbf{x})>0$, define the advantage of $\mathbf{x}$ by 


$$
\left.\left.A d_{l}(\mathbf{x}) \triangleq(E[l(Y, 0) \mid \mathbf{x}])-E[l(Y, 1) \mid \mathbf{x}]\right)\right) P(\mathbf{x})
$$

$A d_{l}(\mathbf{x})>0$ iff $\mathbf{x} \in \mathcal{K}\left[\psi_{\text {opt }}\right]$. An increase in error can arise in two ways from using $\Psi$ instead of $\Psi_{\text {opt }}: \mathbf{x} \in \mathcal{K}\left[\psi_{\text {opt }}\right]$ but $\mathbf{x} \notin \mathcal{K}\left[\psi_{\text {opt }}\right]$, or $\mathbf{x} \notin \mathcal{K}\left[\psi_{\text {opt }}\right]$ but $\mathbf{x} \in \mathcal{K}\left[\psi_{\text {opt }}\right]$. The total error increase from using $\Psi$ instead of $\Psi_{\text {opt }}$ is

$$
\Delta\left(\Psi, \Psi_{\text {oopt }}\right) \triangleq \operatorname{Er}[\Psi]-\operatorname{Er}\left[\Psi_{\text {opt }}\right]=\sum_{\mathbf{x} \in \mathcal{K}\left[\psi_{\text {opt }}\right] \Delta \mathcal{K}[\psi]}\left|A d_{l}(\mathbf{x})\right|
$$

the last sum being over the symmetric difference between the kernels. For MAE, the absolute advantage for $\mathbf{x}$ is given by $|1-2 P(Y=1 \mid \mathbf{x}) P(\mathbf{x})|$.

In practice, the optimal operator is statistically estimated from image realizations. Operator design involves using a random sample $S_{n}$ of pairs $\left(\mathbf{X}^{(1)}, Y^{(1)}\right),\left(\mathbf{X}^{(2)}, Y^{(2)}\right)$ $, \ldots,\left(\mathbf{X}^{(n)}, Y^{(n)}\right)$ to form an estimate $\psi_{n}$ of $\psi_{\text {opt }}$. The error $\operatorname{Er}\left[\psi_{n}\right]$ can not be less than the error $\operatorname{Er}\left[\psi_{\text {opt }}\right]$. Letting $\Delta_{n} \triangleq \operatorname{Er}\left[\psi_{\text {opt }}\right]-\operatorname{Er}\left[\psi_{n}\right]$ denote the design cost (operator estimation error), the error of the designed operator is decomposed as $\operatorname{Er}\left[\psi_{n}\right]=\operatorname{Er}\left[\psi_{\text {opt }}\right]+\Delta_{n}$. Hence, the expected error of the designed operator is $E\left[\operatorname{Er}\left[\psi_{n}\right]\right]=\operatorname{Er}\left[\psi_{\text {opt }}\right]+E\left[\Delta_{n}\right]$. The standard design approach is to estimate the conditional probabilities composing the decision criterion $\left(\psi_{\text {opt }}(\mathbf{x})=1\right.$ if $P(Y=$ $1 \mid \mathbf{x})>0.5)$ and to use these estimates to determine $\psi_{n}$. This method yields a consistent estimate of $\operatorname{Er}\left[\psi_{n}\right]$, that is, $E\left[\Delta_{n}\right] \rightarrow 0$ as $n \rightarrow \infty$.

3.2. Constrained design. Satisfactory image filtering typically requires large windows, and it is often impossible to get large enough samples to sufficiently reduce $E\left[\Delta_{n}\right]$. To ease the design problem, optimization is constrained to some subclass $\mathcal{C}$ of operators. If $\psi_{\mathcal{C}}$ is an optimal operator in $\mathcal{C}$ with error $\operatorname{Er}\left[\psi_{\mathcal{C}}\right]$ and design error $\Delta_{n, \mathcal{C}}$, then $\operatorname{Er}\left[\psi_{\mathcal{C}}\right] \geq \operatorname{Er}\left[\psi_{\text {opt }}\right]$ and $E\left[\Delta_{n, \mathcal{C}}\right] \leq E\left[\Delta_{n}\right]$. The error of a designed constrained operator, $\psi_{n, \mathcal{C}}$, possesses the decomposition $\operatorname{Er}\left[\psi_{n, \mathcal{C}}\right]=\operatorname{Er}\left[\psi_{\mathcal{C}}\right]+\Delta_{n, \mathcal{C}}$. The cost of constraint is given by $\Delta_{\mathcal{C}}=\operatorname{Er}\left[\psi_{\mathcal{C}}\right]-\operatorname{Er}\left[\psi_{\text {opt }}\right]$. Hence, $\operatorname{Er}\left[\psi_{n, \mathcal{C}}\right]=\operatorname{Er}\left[\psi_{\text {opt }}\right]+\Delta_{\mathcal{C}}+\Delta_{n, \mathcal{C}}$, and

$$
E\left[\operatorname{Er}\left[\psi_{n, \mathcal{C}}\right]\right]=\operatorname{Er}\left[\psi_{\text {opt }}\right]+\Delta_{\mathcal{C}}+E\left[\Delta_{n, \mathcal{C}}\right]
$$

A constraint is statistically beneficial iff $E\left[\operatorname{Er}\left[\psi_{n, \mathcal{C}}\right]\right] \leq E\left[\operatorname{Er}\left[\psi_{n}\right]\right]$, which is true iff

$$
\Delta_{\mathcal{C}} \leq E\left[\Delta_{n}\right]-E\left[\Delta_{n, \mathcal{C}}\right]
$$

The saving in design error must exceed the cost of constraint. Since for a consitent estimator $E\left[\Delta_{n}\right]-E\left[\Delta_{n, \mathcal{C}}\right] \rightarrow 0$ as $n \rightarrow \infty$, a constraint can only be beneficial for samples that are not too large. 
A fundamental problem is to find constraints for which $\Delta_{\mathcal{C}} \leq E\left[\Delta_{n}\right]-E\left[\Delta_{n, \mathcal{C}}\right]$. The benefit of a constraint depends on the class of ideal and observed signals under consideration. For instance, suppose a signal is degraded by an extensive operator (the transformed set always includes the input set). Then, letting $\mathcal{C}$ be the class of anti-extensive operators (the transformed set always is included in the input set) yields $\Delta_{\mathcal{C}}=0$, so that there is no constraint error. Such situations rarely occur in practice. Here we describe some constraints that have been studied and proven useful. In many cases the error has been analyzed and quantified; however, we leave that to the literature.

The most studied constraint is that the operator be increasing [12]. Several design methods have been employed to design increasing operators. One way is to find the kernel of the optimal operator and then apply a switching algorithm that derives the optimal increasing operator from the optimal operator by switching structuring elements in and out of the kernel of the designed optimal operator to obtain an increasing operator for which the switching error is minimal [36][25][43]. Once the increasing operator is designed, its basis representation in terms of erosions can be obtained by logically reducing the kernel expansion. A second way to proceed is to estimate the erosion expansion directly. This is achieved by first obtaining MAE estimates for single erosion operators and then recursively obtaining MAE estimates for multiple-erosion operators[31]. The MAE of an $m$-erosion operator $\Psi_{m}$ can be expressed in terms of a single-erosion operator with structuring element $B_{m}$ and two $(m-1)$-erosion operators $\Psi_{m-1}$ and $\Phi_{m-1}$ :

$$
M A E\left[\Psi_{m}\right]=M A E\left[\Psi_{m-1}\right]-M A E\left[\Phi_{m-1}\right]+M A E\left[B_{m}\right],
$$

where the bases are given by $\mathcal{B}\left(\Psi_{m-1}\right)=\left\{B_{1}, B_{2}, \ldots, B_{m-1}\right\}, \mathcal{B}\left(\Psi_{m}\right)=\left\{B_{1}, B_{2}, \ldots, B_{m}\right\}$ and $\mathcal{B}\left(\Phi_{m-1}\right)=\left\{B_{1} \cup B_{m}, B_{2} \cup B_{m}, \ldots, B_{m-1} \cup B_{m}\right\}$. Further constraint can be imposed by limiting the basis size or constraining the search to a subclass of potential structuring elements [30]. Two adaptive procedures have been proposed to design increasing operators. One is based on gradient-type structuring-element adaptation akin to the classical LMS algorithm for linear filters [39]. Another utilizes genetic algorithms to adjust the structuring elements [22]. Comparison of design error for increasing and non-increasing operators has been investigated [17].

One way to classify constraints is to verify whether they are independent or dependent[14]. A constraint is independent if the decision whether to place a vector $\mathbf{x}$ in the kernel is constrained by a condition involving only $\mathbf{x}$ itself, and no other vectors. A dependent constraint is one that cannot be applied to each vector independently. This means there are required relations among vectors that do not reduce to independent constraints. Increasingness is a dependent constraint.

Envelope constraint is an independent constraint that involves two humanly designed operators $\alpha$ and $\beta$, such that $\alpha \leq \beta$. $\mathcal{C}$ is the class of all operators $\psi$ such 
that $\alpha \leq \psi \leq \beta$. Envelope design is a form of human-machine operator design [6]. If the human designed envelope contains the optimal operator, then $\Delta_{\mathcal{C}}=0$; if not, then $\Delta_{\mathcal{C}}>0$ and the constraint is beneficial iff $\Delta_{\mathcal{C}} \leq E\left[\Delta_{n}\right]-E\left[\Delta_{n, \mathcal{C}}\right]$. The key to design is having a sufficiently tight envelope so that design error is reduced with overly increasing constraint error.

In image processing, it is often the case that the variables near the center of the window contribute most to the operator, whereas those on the window periphery contribute less, while enormously increasing the demand for data. In this case, one can apply secondary constraint [41]. The variables near the window center are used in an unconstrained manner, whereas those at the periphery are constrained in how they contribute to the operator. The situation here is that $\Delta_{\mathcal{C}}$ is not too large owing to the lesser importance of peripheral variables in determining $Y$.

Iterative design involves an operator-decomposition constraint. A large window $W$ is decomposed into a Minkowski sum of windows, $W=W_{1} \oplus W_{2} \oplus \ldots \oplus W_{q}$, and $\mathcal{C}$ constrains operators of the form $\Psi=\Psi_{q} \Psi_{q-1} \ldots \Psi_{1}$, where $\psi_{k}$ is defined on $W_{k}$ [40], [26]. Not only do iterative operators require less sample data, they can also possess implementation advantages. $\Delta_{\mathcal{C}}$ depends on the degree to which the optimal operator can be approximated by an iterative operator, relative not only to algebraic decomposition, but also relative to the action of the operator on the random signal process. Iteration can be utilized in conjunction with increasing constraint [20].

Rather than operating on an image at a given resolution, a mapping can be applied to reduce the resolution [15]. While information is lost with resolution reduction, thereby introducing a constraint error, the number of pixels in the window is reduced and this results in smaller design error. One way to take advantage of this is to try to find a resolution at which the optimal operator has minimum error. A better way is, for each observation, decide whether it has been observed sufficiently in training to be confident in the optimal operator at its resolution. If it has been, then apply the operator designed at that resolution; if not, then take lower resolution versions of the vector until it has been observed sufficiently, and then apply an operator designed at an appropriate lower resolution.

Constrained design represents a use of prior information: based on an image degradation model or experience, the cost of constraint is known to be small. Another way to utilize prior knowledge is to begin with a heuristically chosen operator and redefine the kernel if there is sufficient data to indicate a change [7]. Specifically, if $\psi_{\text {pri }}$ is the prior operator, define $\psi_{\text {opt }}(\mathbf{x})=\psi_{\text {pri }}(\mathbf{x})$ unless the estimate of $P(Y=1 \mid \mathbf{x})$ is sufficiently precise to change the definition. The method has been used successfully in digital document processing with the prior operator being the identity, in which case it is known as the method of differencing filters [18].

A more sophisticated form of prior knowledge assumes the conditional probability $P(Y=1 \mid \mathbf{x})$ posses a prior distribution and the data is used to obtain the Bayes 
estimate of $P(Y=1 \mid \mathbf{x})$ relative to the prior distribution [13]. The method is difficult to use owing to the large number of prior distributions required. To help alleviate this problem, it has been used in conjunction with multi-resolution analysis, where its potential effectiveness has been demonstrated [28].

\section{Decomposition of lattice operators}

In the lattice theory, a subset $\mathcal{X}$ of a lattice $(\mathcal{L}, \leq)$ is a sup-generating family for $(\mathcal{L}, \leq)$ if any element of $\mathcal{L}$ is the supremum of elements of $\mathcal{X}$. Dually, $\mathcal{X}$ is an infgenerating family for $(\mathcal{L}, \leq)$ if any element of $\mathcal{L}$ is the infimum of elements of $\mathcal{X}([23]$, p.28).

The concepts of sup- and inf-generating family apply directly to the problem of decomposition of a lattice element in terms of some predefined lattice elements.

We denote by $\bigvee \mathcal{X}$ (resp. $\bigwedge \mathcal{X}$ ) the supremum (resp. infimum) of a subset $\mathcal{X}$ of a lattice $(\mathcal{L}, \leq)$.

One can prove the following general result.

Proposition 5. (decomposition in terms of a sup-generating family) - The subset $\mathcal{X}$ of $\mathcal{L}$ is a sup-generating family for the lattice $(\mathcal{L}, \leq)$ iff, for any $Y$ in $\mathcal{L}$,

$$
Y=\bigvee\{X \in \mathcal{X}: X \leq Y\}
$$

Dually, we have the decomposition in terms of an inf-generating family.

A very nice and now historical example of sup-generating family for a special class of image operators is the Matheron's decomposition ([35], p.219) recalled in Section 2 .

We know that the set of translation invariant increasing operators on binary images is a complete sub-lattice of the binary image operator lattice ([24], Proposition 3.1 ).

Hence, Matheron's decomposition can be stated by saying that the binary image erosions form a sup-generating family for the lattice of translation invariant increasing operators on binary images.

Banon and Barrera's decomposition [2] which is an extension of Matheron's decomposition to non necessarily increasing operators, also recalled in Section 2, provides another example of sup-generating family.

They have proved that the set of Hit-or-Miss operators form a sup-generating family for the lattice of the translation invariant operators on binary images.

In this section, we show that the decomposition of binary image operators, presented in Section 2, is a special case of two types of decomposition of lattice operators (i.e., mappings between complete lattices).

In the next two subsections, we introduce the so-called loose sup-generating operators and then the so-called morphological sup-generating operators. 
4.1. Loose sup-generating operators. Let $\left(\mathcal{L}_{1}, \leq\right)$ and $\left(\mathcal{L}_{2}, \leq\right)$ be two complete lattices. We denote by $O$ and $I$, respectively, the least and greatest elements of these lattices. From now on, we will consider the set of operators from $\mathcal{L}_{1}$ to $\mathcal{L}_{2}$ equipped with the pointwise ordering. This structure is again a complete lattice by inheritance of the complete lattice structure of $\left(\mathcal{L}_{2}, \leq\right)$. It is denoted by $\left(\operatorname{Fun}\left(\mathcal{L}_{1}, \mathcal{L}_{2}\right), \leq\right)$ and called power lattice.

Let $\alpha$ and $\beta$ be two mappings from $\mathcal{L}_{2}$ to $\mathcal{L}_{1}$, we denote by $\overline{\alpha \beta}$ the expression defined by, for any $X$ in $\mathcal{L}_{1}$ :

$$
\overline{\alpha \beta}(X) \triangleq \bigvee\left\{Y \in \mathcal{L}_{2}: \alpha(Y) \leq X \leq \beta(Y)\right\} .
$$

Definition 6. (loose sup-generating operator) - Let $\left(\mathcal{L}_{1}, \leq\right)$ and $\left(\mathcal{L}_{2}, \leq\right)$ be two complete lattices. An operator $\phi$ is a loose sup-generating operator from $\left(\mathcal{L}_{1}, \leq\right)$ to $\left(\mathcal{L}_{2}, \leq\right)$ if there exist two mappings $\alpha$ and $\beta$ from $\mathcal{L}_{2}$ to $\mathcal{L}_{1}$, such that $\phi=\overline{\alpha \beta}$.

The set of loose sup-generating operators is denoted by $\Phi$.

Let us give examples of loose sup-generating operators. Let $U$ and $V$ be two elements, respectively, in $\mathcal{L}_{1}$ and $\mathcal{L}_{2}$, and let $\psi_{(U, V)}$ be the operator from $\mathcal{L}_{1}$ to $\mathcal{L}_{2}$ defined by, for any $X$ in $\mathcal{L}_{1}$,

$$
\psi_{(U, V)}(X) \triangleq\left\{\begin{array}{ll}
V & \text { if } X=U \\
O & \text { otherwise }
\end{array} .\right.
$$

Proposition 7. (examples of loose sup-generating operators) - For any $U$ and $V$, respectively, in $\mathcal{L}_{1}$ and $\mathcal{L}_{2}$, the operator $\psi_{(U, V)}$ is a loose sup-generating operator, that is $\psi_{(U, V)} \in \Phi$.

Proof. $\quad$ Let $U$ and $V$ be two elements, respectively, in $\mathcal{L}_{1}$ and $\mathcal{L}_{2}$, and let $\alpha_{(U, V)}$ and $\beta_{(U, V)}$ be two mappings from $\mathcal{L}_{2}$ to $\mathcal{L}_{1}$ defined by, for any $Y$ in $\mathcal{L}_{2}$,

$$
\alpha_{(U, V)}(Y) \triangleq\left\{\begin{array} { l l } 
{ U } & { \text { if } Y = V } \\
{ I } & { \text { otherwise } }
\end{array} \quad \text { and } \quad \beta _ { ( U , V ) } ( Y ) \triangleq \left\{\begin{array}{ll}
U & \text { if } Y=V \\
O & \text { otherwise }
\end{array} .\right.\right.
$$

For any $U$ and $V$, respectively, in $\mathcal{L}_{1}$ and $\mathcal{L}_{2}$, and any $X$ in $\mathcal{L}_{1}$,

$$
\begin{aligned}
\bigvee\left\{Y \in \mathcal{L}_{2}: \alpha_{(U, V)}(Y) \leq X \leq \beta_{(U, V)}(Y)\right\}= & \bigvee \begin{cases}\{V\} & \text { if } X=U \\
\emptyset & \text { otherwise }\end{cases} \\
& \left(\text { definition of } \alpha_{(U, V)} \text { and } \beta_{(U, V)}\right) \\
= & \begin{cases}V & \text { if } X=U \\
O & \text { otherwise }\end{cases} \\
& (\text { supremum definition }) \\
= & \psi_{(U, V)}(X) \\
& \left(\text { definition of } \psi_{(U, V)}\right) .
\end{aligned}
$$


That is, there exist $\alpha$ and $\beta$, namely $\alpha \triangleq \alpha_{(U, V)}$ and $\beta \triangleq \beta_{(U, V)}$ such that $\psi_{(U, V)}=$ $\overline{\alpha \beta}$, in other words $\psi_{(U, V)} \in \Phi$.

We observe that more than one pair of mappings $\alpha$ and $\beta$ may lead to the same loose sup-generating operator. For example, if $\alpha_{1}(Y)=O$ and $\beta_{1}(Y)=I$ and if the pair of mappings $\alpha_{2}$ and $\beta_{2}$ differs from the pair $\alpha_{1}$ and $\beta_{1}$ just at $Z \leq Y, Z \neq Y$, then we still have $\overline{\alpha_{1} \beta_{1}}=\overline{\alpha_{2} \beta_{2}}$.

The loose sup-generating operators have the following nice property.

Proposition 8. (decomposition in terms of loose sup-generating operators) - The set $\Phi$ of loose sup-generating operators from $\mathcal{L}_{1}$ to $\mathcal{L}_{2}$ is a sup-generating family for the power lattice $\left(\operatorname{Fun}\left(\mathcal{L}_{1}, \mathcal{L}_{2}\right), \leq\right)$. Equivalently, for any $\psi$ in $\operatorname{Fun}\left(\mathcal{L}_{1}, \mathcal{L}_{2}\right)$,

$$
\psi=\bigvee\{\phi \in \Phi: \phi \leq \psi\}
$$

Proof. From Proposition 5 it is sufficient to prove the second assertion. Let us divide the proof in two parts.

i. For any $\psi$ in $\operatorname{Fun}\left(\mathcal{L}_{1}, \mathcal{L}_{2}\right),\{\phi \in \Phi: \phi \leq \psi\}$ has $\psi$ as an upper bound and $\bigvee\{\phi \in \Phi: \phi \leq \psi\}$ as the least upper bound, therefore, we have, $\bigvee\{\phi \in \Phi: \phi \leq \psi\} \leq$ $\psi$.

ii. For any $\psi$ in $\operatorname{Fun}\left(\mathcal{L}_{1}, \mathcal{L}_{2}\right)$ and $U$ in $\mathcal{L}_{1}$, by definition of $\psi_{(U, V)}$ and by Proposition 7, there exists a $\phi \in \Phi$ such that $\phi \leq \psi$ and $\psi(U) \leq \phi(U)$, namely $\phi \triangleq \psi_{(U, V)}$ with $V=\psi(U)$. Therefore, by the supremum definition, for any $U$ in $\mathcal{L}_{1}, \psi(U) \leq$ $\bigvee\{\phi(U): \phi \in \Phi$ and $\phi \leq \psi\}$ that is, by the pointwise ordering definition and the pointwise union definition, $\psi \leq \bigvee\{\phi \in \Phi: \phi \leq \psi\}$

Hence, by the anti-symmetry of $\leq$, we have $\psi=\bigvee\{\phi \in \Phi: \phi \leq \psi\}$.

4.2. Morphological sup-generating operators. We now recall another class of operators ([3], Definition 4.1).

Definition 9. (morphological sup-generating operator) - Let $\left(\mathcal{L}_{1}, \leq\right)$ and $\left(\mathcal{L}_{2}, \leq\right)$ be two complete lattices. An operator $\lambda$ is a morphological sup-generating operator from $\left(\mathcal{L}_{1}, \leq\right)$ to $\left(\mathcal{L}_{2}, \leq\right)$ if $\lambda$ satisfies, for any nonempty subset $\mathcal{X}$ of $\mathcal{L}_{1}$, the following property:

$$
\lambda(\bigwedge \mathcal{X}) \wedge \lambda(\bigvee \mathcal{X})=\bigwedge \lambda(\mathcal{X})
$$


The set of morphological sup-generating operators is denoted by $\Lambda$.

From Proposition 5.5 in [3], we know that any morphological sup-generating mapping is the intersection of an erosion and an anti-dilation.

As it has been shown in [3] (see proof of Lemma 6.1), the operators of the type $\psi_{(U, V)}$ are also examples of morphological sup-generating operators.

As for the loose sup-generating operators, the morphological sup-generating operators have the following nice property.

Proposition 10. (decomposition in terms of morphological sup-generating operators) - The set $\Lambda$ of morphological sup-generating operators from $\mathcal{L}_{1}$ to $\mathcal{L}_{2}$ is a supgenerating family for the power lattice $\left(\operatorname{Fun}\left(\mathcal{L}_{1}, \mathcal{L}_{2}\right), \leq\right)$. Equivalently, for any $\psi$ in $\operatorname{Fun}\left(\mathcal{L}_{1}, \mathcal{L}_{2}\right)$

$$
\psi=\bigvee\{\lambda \in \Lambda: \lambda \leq \psi\}
$$

Proof. See proof of Lemma 6.1 in [3].

At this stage, we have introduced two sup-generating families for the power lattice. We already know that they intersect each other since the operators of the type $\psi_{(U, V)}$ belong to both. Let's be more conclusive.

In order to make the proof of the next proposition simpler to write, we recall, at once, the concept of operator kernel [3].

Let $\psi$ be an operator in $\operatorname{Fun}\left(\mathcal{L}_{1}, \mathcal{L}_{2}\right)$, a mapping $\cdot \mathcal{K}$ from $\operatorname{Fun}\left(\mathcal{L}_{1}, \mathcal{L}_{2}\right)$ to $\operatorname{Fun}\left(\mathcal{L}_{2}, \mathcal{P}\left(\mathcal{L}_{1}\right)\right)$ defined by, for any $Y$ in $\mathcal{L}_{2}$,

$$
\cdot \mathcal{K}(\psi)(Y) \triangleq\left\{X \in \mathcal{L}_{1}: Y \leq \psi(X)\right\} .
$$

is called the left kernel mapping with respect to the complete lattice $\left(\mathcal{L}_{2}, \leq\right)$. The mapping $\cdot \mathcal{K}(\psi)$ from $\mathcal{L}_{2}$ to $\mathcal{P}\left(\mathcal{L}_{1}\right)$ is called the left kernel (or simply, kernel) of $\psi$.

Proposition 11. (comparison between the loose and morphological sup-generating operators) - The set $\Lambda$ of morphological sup-generating operators is included in the set $\Phi$ of loose sup-generating operators, in other words $\Lambda \subset \Phi$.

Proof. Let $\psi$ be the operator from $\mathcal{L}_{1}$ to $\mathcal{L}_{2}$, and let $\underline{\cdot \psi}$ and $\overline{\cdot \psi}$ be the mappings from $\mathcal{L}_{2}$ to $\mathcal{L}_{1}$ defined by, for any $Y$ in $\mathcal{L}_{2}$,

$$
\underline{\psi}(Y) \triangleq \bigwedge \cdot \mathcal{K}(\psi)(Y) \quad \text { and } \quad \bar{\cdot}(Y) \triangleq \bigvee \cdot \mathcal{K}(\psi)(Y)
$$


By Theorem 5.1 in [3], for any $\lambda \in \Lambda$, there exist two mappings $\alpha$ and $\beta$ from $\mathcal{L}_{2}$

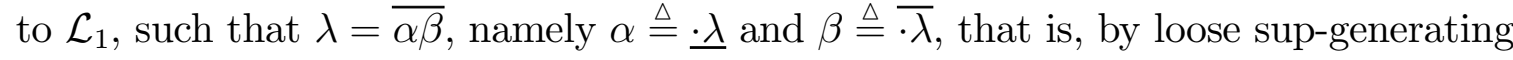
operator definition, $\lambda \in \Phi$, in other words, $\Lambda \subset \Phi$.

Actually, the previous proposition is a consequence of a property of the so-called morphological connection ([3], Definition 5.2). Furthermore, if $\overline{\alpha \beta}$ is a morphological sup-generating operator, then, by Theorem 5.1 in [3], $\alpha$ and $\beta$ are, respectively, a dilation and an anti-dilation. This means that $\alpha$ is necessarily increasing and $\beta$ is necessarily decreasing.

We have chosen to qualify the sup-generating operators in $\Phi$ as loose because they don't necessarily have to be derived from an increasing and a decreasing mappings. Even though two inputs are comparable, in the loose case, the $\alpha$ and $\beta$ outputs don't need to be comparable

We have chosen to qualify the sup-generating operators in $\Lambda$ as morphological because they are derived from a dilation and an anti-dilation which are two elementary morphological mappings.

At first glance, one could think that the morphological decomposition is better than the loose one in the sense that we don't need to consider all the loose sup-generating operators to construct any lattice operator; the morphological supgenerating operators are sufficient.

Nevertheless, one can expect that more morphological than loose sup-generating operators may be needed in a minimal construction.

4.3. Constructive decompositions. In order to state the constructive decomposition of a lattice operator, we have to recall the concept of interval function ([3], Definition 6.2).

$\mathcal{I}$ is an interval function from $\mathcal{L}_{2}$ to $\mathcal{P}\left(\mathcal{L}_{1}\right)$ if for any $Y$ in $\mathcal{L}_{2}, \mathcal{I}(Y)$ is a interval of $\mathcal{P}\left(\mathcal{L}_{1}\right)$ or the empty set. We denote by $\operatorname{IntFun}\left(\mathcal{L}_{2}, \mathcal{P}\left(\mathcal{L}_{1}\right)\right)$ the set of interval functions from $\mathcal{L}_{2}$ to $\mathcal{P}\left(\mathcal{L}_{1}\right)$.

We denote by $\cdot \mathbf{A}_{1}(\psi)$ the interval function collection defined by, for any $\psi$ from $\mathcal{L}_{1}$ to $\mathcal{L}_{2}$,

$$
\cdot \mathbf{A}_{1}(\psi) \triangleq\left\{\mathcal{I} \in \operatorname{IntFun}\left(\mathcal{L}_{2}, \mathcal{P}\left(\mathcal{L}_{1}\right)\right): \mathcal{I} \leq \cdot \mathcal{K}(\psi)\right\} .
$$

In the above statement, $\leq$ is the pointwise ordering on $\operatorname{Fun}\left(\mathcal{L}_{2}, \mathcal{P}\left(\mathcal{L}_{1}\right)\right)$, that is the extension of the inclusion $\subseteq$ on $\mathcal{P}\left(\mathcal{L}_{1}\right)$ to $\operatorname{Fun}\left(\mathcal{L}_{2}, \mathcal{P}\left(\mathcal{L}_{1}\right)\right)$.

Let $\mathcal{I}$ be an interval function from $\mathcal{L}_{2}$ to $\mathcal{P}\left(\mathcal{L}_{1}\right)$, we denote by $\alpha_{\mathcal{I}}$ and $\beta_{\mathcal{I}}$ the mappings from $\mathcal{L}_{2}$ to $\mathcal{L}_{1}$ defined by, for any $Y$ in $\mathcal{L}_{2}$,

$$
\alpha_{\mathcal{I}}(Y) \triangleq \bigwedge \mathcal{I}(Y) \quad \text { and } \quad \beta_{\mathcal{I}}(Y) \triangleq \bigvee \mathcal{I}(Y)
$$


We call the mappings $\alpha_{\mathcal{I}}$ and $\beta_{\mathcal{I}}$ the extremity functions of $\mathcal{I}$. For any $Y$ in $\mathcal{L}_{2}$, we have $\alpha_{\mathcal{I}}(Y) \leq \beta_{\mathcal{I}}(Y)$ or (exclusive) $\left(\alpha_{\mathcal{I}}(Y)=I\right.$ and $\left.\beta_{\mathcal{I}}(Y)=O\right)$.

By observing that for any $X$ in $\mathcal{L}_{1}, \psi(X)=\bigvee\left\{Y \in \mathcal{L}_{2}: Y \leq \psi(X)\right\}$, we can prove the following theorem.

Theorem 12. (constructive decomposition in terms of loose sup-generating operators) - Let $\left(\mathcal{L}_{1}, \leq\right)$ and $\left(\mathcal{L}_{2}, \leq\right)$ be two lattices. Any operator $\psi$ from $\mathcal{L}_{1}$ to $\mathcal{L}_{2}$ can be decomposed by a set of loose sup-generating operators and its constructive decomposition is

$$
\psi=\bigvee\left\{\overline{\alpha_{\mathcal{I}} \beta_{\mathcal{I}}}: \mathcal{I} \in \cdot \mathbf{A}_{1}(\psi)\right\}
$$

Let $\Delta$ and $\Delta^{\mathrm{a}}$ be, respectively, the set of dilations and anti-dilations from $\mathcal{L}_{2}$ to $\mathcal{L}_{1}$.

We denote by $\cdot \mathbf{A}_{\mathrm{m}}(\psi)$ the interval function collection defined by, for any $\psi$ from $\mathcal{L}_{1}$ to $\mathcal{L}_{2}$,

$$
\cdot \mathbf{A}_{\mathrm{m}}(\psi) \triangleq\left\{\mathcal{I} \in \operatorname{IntFun}\left(\mathcal{L}_{2}, \mathcal{P}\left(\mathcal{L}_{1}\right)\right): \mathcal{I} \leq \cdot \mathcal{K}(\psi), \alpha_{\mathcal{I}} \in \Delta \text { and } \beta_{\mathcal{I}} \in \Delta^{\mathrm{a}}\right\}
$$

When using only the morphological sup-generating operators, we have the following theorem.

Theorem 13. (constructive decomposition in terms of morphological sup-generating operators $)$ - Let $\left(\mathcal{L}_{1}, \leq\right)$ and $\left(\mathcal{L}_{2}, \leq\right)$ be two lattices. Any operator $\psi$ from $\mathcal{L}_{1}$ to $\mathcal{L}_{2}$ can be decomposed by a set of morphological sup-generating operators and its constructive decomposition is

$$
\psi=\bigvee\left\{\overline{\alpha_{\mathcal{I}} \beta_{\mathcal{I}}}: \mathcal{I} \in \cdot \mathbf{A}_{\mathrm{m}}(\psi)\right\}
$$

Proof. See proof of Theorem in [3].

We now make a comparison between the loose and morphological sup-generating operators in terms of their kernels.

Let $\alpha$ and $\beta$ be two mappings from $\mathcal{L}_{2}$ to $\mathcal{L}_{1}$, we denote by $[\alpha, \beta]$ the interval function from $\mathcal{L}_{2}$ to $\mathcal{P}\left(\mathcal{L}_{1}\right)$ defined by, for any $Y$ in $\mathcal{L}_{2}$, 


$$
[\alpha, \beta](Y) \triangleq \begin{cases}{[\alpha(Y), \beta(Y)]} & \text { if } \alpha(Y) \leq \beta(Y) \\ \emptyset & \text { otherwise }\end{cases}
$$

From the definitions of $\overline{\alpha \beta}$ and morphological connection ([3], Proposition 5.2), we can prove the following proposition.

Proposition 14. (loose and morphological sup-generating operator kernel properties) - We have the following properties.

i) Let $\psi$ be a loose sup-generating operator from $\left(\mathcal{L}_{1}, \leq\right)$ to $\left(\mathcal{L}_{2}, \leq\right)$, and let $\alpha$ and $\beta$ be two mappings from $\mathcal{L}_{2}$ to $\mathcal{L}_{1}$, such that $\psi=\overline{\alpha \beta}$, then $[\alpha, \beta] \leq \cdot \mathcal{K}(\psi)$.

ii) Let $\psi$ be an operator from $\mathcal{L}_{1}$ to $\mathcal{L}_{2}$, and let $\alpha \triangleq \cdot \psi$ and $\beta \triangleq \bar{\cdot}$, then $\mathcal{K}(\psi) \leq[\alpha, \beta]$. Furthermore, $\psi$ is a morphological sup-generating operator from $\left(\mathcal{L}_{1}, \leq\right)$ to $\left(\mathcal{L}_{2}, \leq\right)$ if and only if $\cdot \mathcal{K}(\psi)=[\alpha, \beta]$.

The above proposition shows that the equality holds for and only for the morphological sup-generating operators.

4.4. Minimal decompositions. Since $\cdot \mathbf{A}_{\mathrm{m}}(\psi)$ is included in $\cdot \mathbf{A}_{l}(\psi)$, the morphological decomposition is slimmer than the loose one. Nevertheless, when the decompositions are based on the maximal interval functions of $\cdot \mathbf{A}_{1}(\psi)$ and $\cdot \mathbf{A}_{\mathrm{m}}(\psi)$, then we may come to the opposite conclusion.

For the sake of simplicity, we will assume, from now on, that the sets $\mathcal{L}_{1}$ and $\mathcal{L}_{2}$ are finite. We denote by $\operatorname{Max} \mathcal{A}$ the set of maximal elements of a subset $\mathcal{A}$ of a lattice.

Let $\cdot \mathbf{B}_{\mathrm{l}}(\psi)$ and $\cdot \mathbf{B}_{\mathrm{m}}(\psi)$ be, respectively, the collection of maximal interval functions of $\cdot \mathbf{A}_{\mathrm{l}}(\psi)$ and $\cdot \mathbf{A}_{\mathrm{m}}(\psi)$, that is, $\cdot \mathbf{B}_{\mathrm{l}}(\psi)=\operatorname{Max} \mathbf{A}_{\mathrm{l}}(\psi)$ and $\cdot \mathbf{B}_{\mathrm{m}}(\psi)=\operatorname{Max} \cdot \mathbf{A}_{\mathrm{m}}(\psi)$. We call them, respectively, the loose (left) basis and the morphological (left) basis of $\psi$.

For any $\mathcal{I}$ and $\mathcal{J}$ in $\operatorname{IntFun}\left(\mathcal{L}_{2}, \mathcal{P}\left(\mathcal{L}_{1}\right)\right), \mathcal{I} \leq \mathcal{J}$ implies $\overline{\alpha_{\mathcal{I}} \beta_{\mathcal{I}}} \leq \overline{\alpha_{\mathcal{J}} \beta_{\mathcal{J}}}$. Hence if $\mathcal{I}$ and $\mathcal{J}$ are two interval functions in $\cdot \mathbf{A}_{1}(\psi)$ (resp., $\left.\cdot \mathbf{A}_{\mathrm{m}}(\psi)\right)$ and $\mathcal{I} \leq \mathcal{J}$, then $\overline{\alpha_{\mathcal{I}} \beta_{\mathcal{I}}}$ has no contribution in the constructive loose (resp., morphological) decomposition of $\psi$. Therefore, in the finite case, we have the following two theorems.

Theorem 15. (minimal decomposition in terms of loose sup-generating operators) Let $\left(\mathcal{L}_{1}, \leq\right)$ and $\left(\mathcal{L}_{2}, \leq\right)$ be two finite lattices. Any operator $\psi$ from $\mathcal{L}_{1}$ to $\mathcal{L}_{2}$ can be decomposed by a set of loose sup-generating operators and its minimal decomposition is

$$
\psi=\bigvee\left\{\overline{\alpha_{\mathcal{I}} \beta_{\mathcal{I}}}: \mathcal{I} \in \cdot \mathbf{B}_{1}(\psi)\right\}
$$


Theorem 16. (minimal decomposition in terms of morphological sup-generating operators) - Let $\left(\mathcal{L}_{1}, \leq\right)$ and $\left(\mathcal{L}_{2}, \leq\right)$ be two finite lattices. Any operator $\psi$ from $\mathcal{L}_{1}$ to $\mathcal{L}_{2}$ can be decomposed by a set of morphological sup-generating operators and its minimal decomposition is

$$
\psi=\bigvee\left\{\overline{\alpha_{\mathcal{I}} \beta_{\mathcal{I}}}: \mathcal{I} \in \cdot \mathbf{B}_{\mathrm{m}}(\psi)\right\}
$$

The minimal loose decomposition can still be slimmed since it may involve redundant loose sup-generating operators:

$$
\psi=\bigvee \operatorname{Max}\left\{\overline{\alpha_{\mathcal{I}} \beta_{\mathcal{I}}}: \mathcal{I} \in \cdot \mathbf{B}_{1}(\psi)\right\}
$$

For any operator $\psi$ in $\operatorname{Fun}\left(\mathcal{L}_{1}, \mathcal{L}_{2}\right)$, we denote by $\cdot \mathbf{B}(\psi)$ the mapping from $\mathcal{L}_{2}$ to $\mathcal{P}\left(\mathcal{P}\left(\mathcal{L}_{1}\right)\right)$ such that, for any $Y$ in $\mathcal{L}_{2}, \cdot \mathbf{B}(\psi)(Y)$ is the subcollection of maximal intervals of $\cdot \mathcal{K}(\psi)(Y)$, that is, $\cdot \mathbf{B}(\psi)(Y)=\operatorname{Max} \cdot \mathcal{K}(\psi)(Y)$

We write $\mathcal{I}(\in) \cdot \mathbf{B}(\psi)$ to mean that, for any $Y$ in $\mathcal{L}_{2}, \mathcal{I}(Y) \in \cdot \mathbf{B}(\psi)(Y)$. We can prove that $\mathcal{I}(\in) \cdot \mathbf{B}(\psi)$ is equivalent to $\mathcal{I} \in \cdot \mathbf{B}_{l}(\psi)$ and consequently, for any $X$ in $\mathcal{L}_{1}$,

$$
\psi(X)=\bigvee\left\{Y \in \mathcal{L}_{2}: \exists I \in \cdot \mathbf{B}(\psi)(Y): X \in I\right\}
$$

This expression, derived from the minimal loose decomposition, is attractive because it is computationally simple. It has been extensively used in [10] and [5].

When $\mathcal{L}_{2}$ is simply a chain with two elements then the minimal loose decomposition becomes a minimal morphological decomposition. This is a consequence of the fact that, in this case, for any $\mathcal{I} \in \cdot \mathbf{B}_{1}(\psi), \alpha_{\mathcal{I}} \in \Delta$ and $\beta_{\mathcal{I}} \in \Delta^{\mathrm{a}}$.

In other words, the decomposition of binary image operators is a special case of the two decompositions presented above.

\section{GRAY-SCALE OPERATOR DESign}

Optimizing gray-scale operators is inherently more difficult than optimizing binary operators owing to the much greater complexity of gray-scale characteristic functions; nevertheless the problem has been addressed, for quite some time, for increasing operators, and more recently for non-increasing operators [19]. Owing to space limitations, we will confine ourselves to brief descriptions of the kind of gray-scale designs that have been studied. From the standpoint of representation, the lattice representations are again involved, with lattices being discrete-integer valued rather than binary. 
The optimization problem for increasing gray-scale operators has been studied in several ways. Among the first issue addressed was finding the maximal search space for the structuring elements. Later, MAE theorems were derived in different settings, and these were used in much the same way as the MAE theorem in the binary case [31], [32]. The gradient-type adaptation mentioned earlier for binary operators has also been applied.

A constrained class of increasing operators are those that satisfy the threshold decomposition property. For any random variable $X$, define the binary random variable $X^{k}$ by $X^{k} \triangleq 1$ if $X \geq k$ and $X^{k} \triangleq 0$ if $X<k$. $\psi$ satisfies the threshold decomposition property if there exists an increasing binary operator $\zeta_{\psi}$ such that

$$
\psi(\mathbf{X})=\sum_{k=1}^{m} \zeta_{\psi}\left(\mathbf{X}^{k}\right)
$$

where $\mathbf{X} \triangleq\left(X_{1}, X_{2}, \ldots, X_{d}\right)$ and $\mathbf{X}^{k} \triangleq\left(X_{1}^{k}, X_{2}^{k}, \ldots, X_{d}^{k}\right)$. Since $\psi$ is defined via a single binary operator, it is in effect a binary operator. Its basis representation consists of erosions with flat structuring elements. Thus, such filters are called flat filters or stack filters. The design cost for flat filters is reduced in comparison to general increasing filters, but the constraint error is increased. The increase is sufficiently severe in most cases that flat filters are essentially confined to restoring signals degraded by additive noise. In operator design it is assumed that all threshold vectors $\mathbf{X}^{k}$ are identically distributed. Numerous design methods have been developed for flat filters [11], [1], [21], [29].

Up till recently the statistical design of non-increasing operators had been kept to very small windows or to operators that are combinations of increasing operators. The opportunities have been expanded with the introduction of aperture filters [27]. These are windowed in both domain and range, thereby reducing design complexity resulting from the gray-scale. Owing to reduced design error and larger windows, aperture filters have been shown to outperform unconstrained gray-scale operators. Indeed, they have been shown to outperform linear inverse (Wiener) filters for restoring images corrupted by nonlinear smoothing [27]. Active research on aperture filters is continuing, with various special types of aperture filters under investigation.

\section{Conclusion}

The study of the morphological language, initiated by Matheron in the seventies, has led to the construction of a solid algebraic framework for the design of non-linear discrete signal and image processing operators. Compared to other algebraic structures as neural networks or decision trees, this approach has an important advantage: the adequacy for modeling prior information (i.e., user's knowledge about the problem studied). This point is absolutely fundamental for practical application of the 
technique, otherwise the amount of training data necessary would be prohibitive. Examples of techniques for modeling prior information in morphological operators design are envelopes, multi-resolution and apertures. These approaches have been successfully applied in the design of document image processing procedures.

Usually, the basis estimation algorithms construct the basis components from which can be derived the loose canonical representation. However, this canonical representation may not be efficient for computing the operators. Trying to overcome this difficulty, recent studies present techniques for the transformation of some canonical morphological representations into more efficient morphological operators [38], [9], [37]. Even in the simple cases studied, these techniques require the solution of complex combinatorial optimization problems.

There are many challenges for future work in this field, mainly in relation to modeling of prior knowledge and to creating specialized basis estimation algorithms. Perhaps the greatest future challenge is the generalization of theese ideas to the identification of discrete-time discrete-range dynamical systems. This advance would have many applications in the modeling of genetic networks from observation of expression patterns by microarray technology, which is among the greatest scientific challenges of the twenty-first century.

\section{REFERENCES}

[1] J. T. Astola and P. Kuosmanen. Representation and optimization of stack filters. In E. R. Dougherty and J. T. Astola, editors, Nonlinear Filters for Image Processing, pages 237-279. SPIE and IEEE Press, Bellingham, 1999.

[2] G. J. F. Banon and J. Barrera. Minimal Representations for TranslationInvariant Set Mappings by Mathematical Morphology. SIAM J. Applied Mathematics, 51(6):1782-1798, December 1991.

[3] G. J. F. Banon and J. Barrera. Decomposition of Mappings between Complete Lattices by Mathematical Morphology, Part I. General Lattices. Signal Processing, 30:299-327, 1993.

[4] J. Barrera and G. J. F. Banon. Expressiveness of the Morphological Language. In Image Algebra and Morphological Image Processing III, volume 1769 of Proc. of SPIE, pages 264-274, San Diego, California, 1992.

[5] J. Barrera and E. R. Dougherty. Representation of Gray-Scale Windowed Operators. In H. J. Heijmans and J. B. Roerdink, editors, Mathematical Morphology and its Applications to Image and Signal Processing, volume 12 of Computational Imaging and Vision, pages 19-26. Kluwer Academic Publishers, Dordrecht, May 1998. 
[6] J. Barrera, E. R. Dougherty, and M. Brun. Hybrid human-machine binary morphological operator design. An independent constraint approach. Signal Processing, 80(8):1469-1487, August 2000.

[7] J. Barrera, E. R. Dougherty, and N. S. T. Hirata. Design of Optimal Morphological Operators from Prior Filters. Acta Steriologica, 16(3):193-200, 1997. Special issue on Mathematical Morphology.

[8] J. Barrera, E. R. Dougherty, and N. S. Tomita. Automatic Programming of Binary Morphological Machines by Design of Statistically Optimal Operators in the Context of Computational Learning Theory. Electronic Imaging, 6(1):54-67, January 1997.

[9] J. Barrera and R. F. Hashimoto. Sup-compact and inf-compact representation of w-operators. Fundamenta Informaticae, 45(4):283-294, 2001.

[10] J. Barrera, R. Terada, R. Hirata Jr, and N. S. T. Hirata. Automatic Programming of Morphological Machines by PAC Learning. Fundamenta Informaticae, 41(1-2):229-258, January 2000.

[11] E. J. Coyle and J.-H. Lin. Stack Filters and the Mean Absolute Error Criterion. IEEE Transactions on Acoustics, Speech and Signal Processing, 36(8):1244-1254, August 1988.

[12] E. R. Dougherty. Optimal Mean-Square N-Observation Digital Morphological Filters I. Optimal Binary Filters. CVGIP: Image Understanding, 55(1):36-54, January 1992.

[13] E. R. Dougherty and J. Barrera. Bayesian Design of Optimal Morphological Operators Based on Prior Distributions for Conditional Probabilities. Acta Stereologica, 16(3):167-174, 1997.

[14] E. R. Dougherty and J. Barrera. Logical Image Operators. In E. R. Dougherty and J. T. Astola, editors, Nonlinear Filters for Image Processing, pages 1-60. SPIE and IEEE Press, Bellingham, 1999.

[15] E. R. Dougherty, J. Barrera, G. Mozelle, S. Kim, and M. Brun. Multiresolution Analysis for Optimal Binary Filters. Mathematical Imaging and Vision, (14):53$72,2001$.

[16] E. R. Dougherty and C. R. Giardina. A digital version of the matheron representation theorem for increasing tau-mappings in terms of a basis for the kernel. In Proc. IEEE Computer Vision and Pattern Recognition, pages 534-536, Miami, 1986. 
[17] E. R. Dougherty and R. P. Loce. Precision of Morphological-Representation Estimators for Translation-invariant Binary Filters: Increasing and Nonincreasing. Signal Processing, 40:129-154, 1994.

[18] E. R. Dougherty and R. P. Loce. Optimal Binary Differencing Filters: Design, Logic Complexity, Precision Analysis, and Application to Digital Document Processing. Electronic Imaging, 5(1):66-86, January 1996.

[19] E. R. Dougherty and D. Sinha. Computational Mathematical Morphology. Signal Processing, 38:21-29, 1994.

[20] E. R. Dougherty, Y. Zhang, and Y. Chen. Optimal Iterative Increasing Binary Morphological Filters. Optical Engineering, 35(12):3495-3507, December 1996.

[21] M. Gabbouj and E. J. Coyle. Minimum Mean Absolute Error Stack Filtering with Structural Constraints and Goals. IEEE Transactions on Acoustics, Speech and Signal Processing, 38(6):955-968, June 1990.

[22] N. R. Harvey and S. Marshall. The Use of Genetic Algorithms in Morphological Filter Design. Signal Processing: Image Communication, 8(1):55-71, January 1996.

[23] H. J. A. M. Heijmans. Morphological Image Operators. Academic Press, Boston, 1994.

[24] H. J. A. M. Heijmans and C. Ronse. The Algebraic Basis of Mathematical Morphology - Part I: Dilations and Erosions. Computer Vision, Graphics and Image Processing, 50:245-295, 1990.

[25] N. S. T. Hirata, E. R. Dougherty, and J. Barrera. A Switching Algorithm for Design of Optimal Increasing Binary Filters Over Large Windows. Pattern Recognition, 33(6):1059-1081, June 2000.

[26] N. S. T. Hirata, E. R. Dougherty, and J. Barrera. Iterative Design of Morphological Binary Image Operators. Optical Engineering, 39(12):3106-3123, December 2000.

[27] R. Hirata Jr., E. R. Dougherty, and J. Barrera. Aperture Filters. Signal Processing, 80(4):697-721, April 2000.

[28] V. G. Kamat, E. R. Dougherty, and J. Barrera. Multiresolution Bayesian Design of Binary Filters. submitted, 2000. 
[29] P. Kuosmanen and J. T. Astola. Optimal stack filters under rank selection and structural constraints. Signal Processing, 41:309-338, 1995.

[30] R. P. Loce and E. R. Dougherty. Facilitation of Optimal Binary Morphological Filter Design Via Structuring Element Libraries and Design Constraints. Optical Engineering, 31(5):1008-1025, May 1992.

[31] R. P. Loce and E. R. Dougherty. Optimal Morphological Restoration: The Morphological Filter Mean-Absolute-Error Theorem. Visual Communication and Image Representation, 3(4):412-432, December 1992.

[32] R. P. Loce and E. R. Dougherty. Mean-Absolute-Error representation and Optimization of Computational-Morphological Filters. Graphical Models and Image Processing, 57(1):27-37, 1995.

[33] P. Maragos. A Representation Theory for Morphological Image and Signal Processing. IEEE Transactions on Pattern Analysis and Machine Intelligence, 11(6):586-599, June 1989.

[34] P. A. Maragos. A Unified Theory of Translation-invariant Systems with Applications to Morphological Analysis and Coding of Images. PhD thesis, School of Elect. Eng. - Georgia Inst. Tech., 1985.

[35] G. Matheron. Random Sets and Integral Geometry. John Wiley, 1975.

[36] A. V. Mathew, E. R. Dougherty, and V. Swarnakar. Efficient Derivation of the Optimal Mean-Square Binary Morphological Filter from the Conditional Expectation Via a Switching Algorithm for Discrete Power-Set Lattice. Circuits, Systems, and Signal Processing, 12(3):409-430, 1993.

[37] J. B. R. F. Hashimoto and E. R. Dougherty. From the sup-decomposition to a sequential decomposition. In L. V. John Goutsias and D. S. Bloomberg, editors, Mathematical morphology and its applications to image and signal processing, pages 13-22, Palo Alto, 2000.

[38] J. B. R. F. Hashimoto and C. E. Ferreira. A combinatorial optimization technique for the sequential decomposition of erosions and dilations. Journal of Mathematical Imaging and Vision, 13(1):17-33, 2000.

[39] P. Salembier. Structuring element adaptation for morphological filters. Visual Communication and Image Representation, 3(2):115-136, 1992.

[40] O. V. Sarca, E. Dougherty, and J. Astola. Two-stage Binary Filters. Electronic Imaging, 8(3):219-232, July 1999. 
[41] O. V. Sarca, E. R. Dougherty, and J. Astola. Secondarily Constrained Boolean Filters. Signal Processing, 71(3):247-263, December 1998.

[42] J. Serra. Image Analysis and Mathematical Morphology. Academic Press, 1982.

[43] I. Tăbuş, D. Petrescu, and M. Gabbouj. A training Framework for Stack and Boolean Filtering - Fast Optimal Design Procedures and Robustness Case Study. IEEE Transactions on Image Processing, 5(6):809-826, June 1996. 